\title{
Surgical Management of an Open Apex with Platelet-Rich Fibrin and Hydroxyapatite Bone Graft - A Case Report
}

\author{
Rajakeerthi R. ${ }^{1}$, Nivedhitha M. S. ${ }^{2}$, Madhumita S. ${ }^{3}$, Dhanavel Chakravarthy ${ }^{4}$ \\ 1,3,4 Department of Conservative Dentistry \& Endodontics, Indira Gandhi Institute of Dental Sciences, Sri Balaji \\ Vidyapeeth University, Pillaiyarkuppam, Pondicherry, India. ${ }^{2}$ Department of Conservative Dentistry \& Endodontics, \\ Saveetha Dental College, Saveetha Institute of Medical and Technical Sciences, Chennai, India.
}

\section{INTRODUCTION}

Periapical surgery is the treatment of choice for a large periapical lesion with bone loss that occurs due to long standing untreated teeth affected by trauma, caries or in situations where there is an endodontic treatment failure. Failure of nonsurgical endodontic treatment may be due to peri radicular tissues incorporating with infections, foreign body response to filling materials, over instrumentation or over obturation which might have prevented complete periapical healing. We present here 2 case reports wherein, combination of platelet-rich fibrin (PRF) and the hydroxyapatite (HA) graft was used to achieve rapid healing of large periapical lesions, and the treatment outcome concluded that PRF and hydroxyapatite bone graft accelerated the wound healing and induced the rapid rate of bone formation, which was confirmed radiographically.

Untreated non-vital teeth generally result in bone destruction in periapical region, which may occur due to the spread of infection following pulpal necrosis, trauma or failed endodontic treatment. Surgical intervention is essential when endodontic treatment has failed and retreatment is not possible. Periapical surgery, Apicoectomy or root end resection endodontic surgery is a safe and well documented treatment alternative when teeth with periapical pathosis are not responding to conventional endodontic treatment. ${ }^{1}$ Periapical surgery removes periapical pathosis resulting in optimal wound healing with regeneration of the bone and periodontal tissue.

Regeneration has been defined as the reproduction or reconstitution of a lost or injured part to restore their architecture and function. The nature of wound healing and regeneration after endodontic surgery depends on the availability of progenitor cells, signalling molecules and micro environmental cues. ${ }^{2}$ The need of a biological modulator to enhance the healing after such surgeries lead to development of platelet rich plasma (PRP) by Whitman et al. in 1997. PRP is an autologous concentrate of platelets suspended in plasma. Subsequently, Choukroun et al. formulated, platelet rich fibrin (PRF) which is a second generation of platelet concentrate. The major advantage of PRF is the ease of preparation and application. It is less expensive and requires less biochemical modifications. ${ }^{1}$

After surgical intervention, healing occurs by formation of connective tissue, which is not desired from the endodontist's point of view. PRF is both a healing and an interpositional biomaterial which acts as a competitive barrier between desired and undesired cells and prevents proliferating of connective tissue of the oral epithelium and gingiva from growing into the defect. ${ }^{3}$ PRF contains growth factors such as platelet derived growth factor (PDGF), transforming growth factor $\beta 1$ (TGF $\beta 1$ ), insulin like growth factor (IGF). It procures properties like cell migration, cell attachment, cell proliferation, and cell differentiation. ${ }^{3,2}$
Corresponding Author: Dr. Madhumita Srinivasan, Post Graduate,

Department of Conservative Dentistry \& Endodontics, Indira Gandhi Institute of Dental Sciences, Sri Balaji Vidyapeeth University, Pillaiyarkuppam,

Pondicherry, India.

E-mail:

dr.madhumitasrinivasan1571995@gmail.c om

\section{DOI: $10.14260 / j e m d s / 2021 / 478$}

How to Cite This Article:

Rajakeerthi R, Nivedhitha MS, Madhumita $S$, et al. Surgical management of an open apex with platelet-rich fibrin and hydroxyapatite bone graft - a case report. J Evolution Med Dent Sci 2021;10(30):23382342, DOI: $10.14260 /$ jemds/2021/478

Submission 09-06-2020,

Peer Review 05-07-2021,

Acceptance 12-07-2021,

Published 26-07-2021.

Copyright (C) 2021 Rajakeerthi R. et al. This is an open access article distributed under Creative Commons Attribution License [Attribution 4.0 International (CC BY 4.0)] 
Using PRF with autogenous bone graft helps to accelerate the body's natural wound healing mechanisms and regeneration by utilizing the patient's own blood. ${ }^{3}$ Hydroxyapatite is one of the bioceramics and is a regenerative graft material which is placed in the bony defect. It causes bone regeneration by conduction or by acting as a scaffold and the defect is filled. Literature has reported the osteoinductive property of "HA" which is highly promising for new bone regeneration. ${ }^{4}$ The purpose of this case report is to evaluate the use of platelet rich fibrin (PRF) used along with hydroxyapatite bone graft in the treatment of large periapical lesion.

\section{PRESENTATION OF CASES}

\section{Case Report 1}

A young adolescent 18-year-old female patient reported to the Department of Conservative Dentistry and Endodontics with a chief complaint of discoloration in relation to upper front teeth for the past 1 year. The history revealed that she had a fall before 2 years and had broken her maxillary anterior teeth. The swelling was initially small, then gradually progressed and was associated with discomfort while taking food. On Intraoral examination 11,12 were fractured (Ellis class IV fracture) and discoloured. On palpation there was tenderness over the apical mucosa in relation to 11,12 and there was pain on percussion. A pulp sensitivity test revealed that 11,12 were non-vital. Preoperative intraoral periapical radiograph of 11 , 12 revealed the presence of large irregular periapical radiolucency ( $4 \mathrm{~cm} \times 3 \mathrm{~cm}$ in size) at the apex of 11, 12 (Fig. 1a).
Access opening was done in both teeth, working length was determined and a thorough chemo mechanical preparation was performed. The pulp chamber and root canals were packed with calcium hydroxide paste until the paste was seen at the canal orifice. After 2 months follow up, radiographic examination revealed the persistence of the periapical lesion. The case was evaluated and treatment plan was decided taking into consideration the history, clinical examination and radio graphic interpretation. Surgical intervention was planned and the patient was explained about the treatment plan. A cone beam computed tomography (CBCT) scan a (DENTSPLY Sirona3D (orthophos XG 3D) was advised. (Fig.1c \& 1d).

The non-surgical endodontic procedure was performed by using step back technique till the apical size was \#50, \#55, \#45, and \#60 with regard to teeth 11,12 respectively (Fig. 2a \& 2b). Sodium Hypochlorite (5.25 \%) solution was used for irrigation during the canal preparation. Gutta percha (Dentsply Maillefer) was used for canal obturation and AH 26 sealer (DENTSPLY) was used by roll cone technique. Before the surgical intervention, basic blood investigations were carried out and the results were within the physiological limits. Informed consent was also obtained from the patient.

Under local anaesthesia (1:200,000 adrenaline). Sulcular incision starting from the distal of the maxillary right central incisor to mesial of the maxillary right canine was made, a full - thickness mucoperiosteal flap was raised, the surgical site revealed mild loss of labial cortical plate (Fig 4a).

The periapical defect was curetted, and the diseased tissue was totally removed and irrigated. Biodentine apical barrier was placed in single step by placing $4-5 \mathrm{~mm}$ Biodentine (septodont) apical plug, using Schilder pluggers. (Fig 4b)

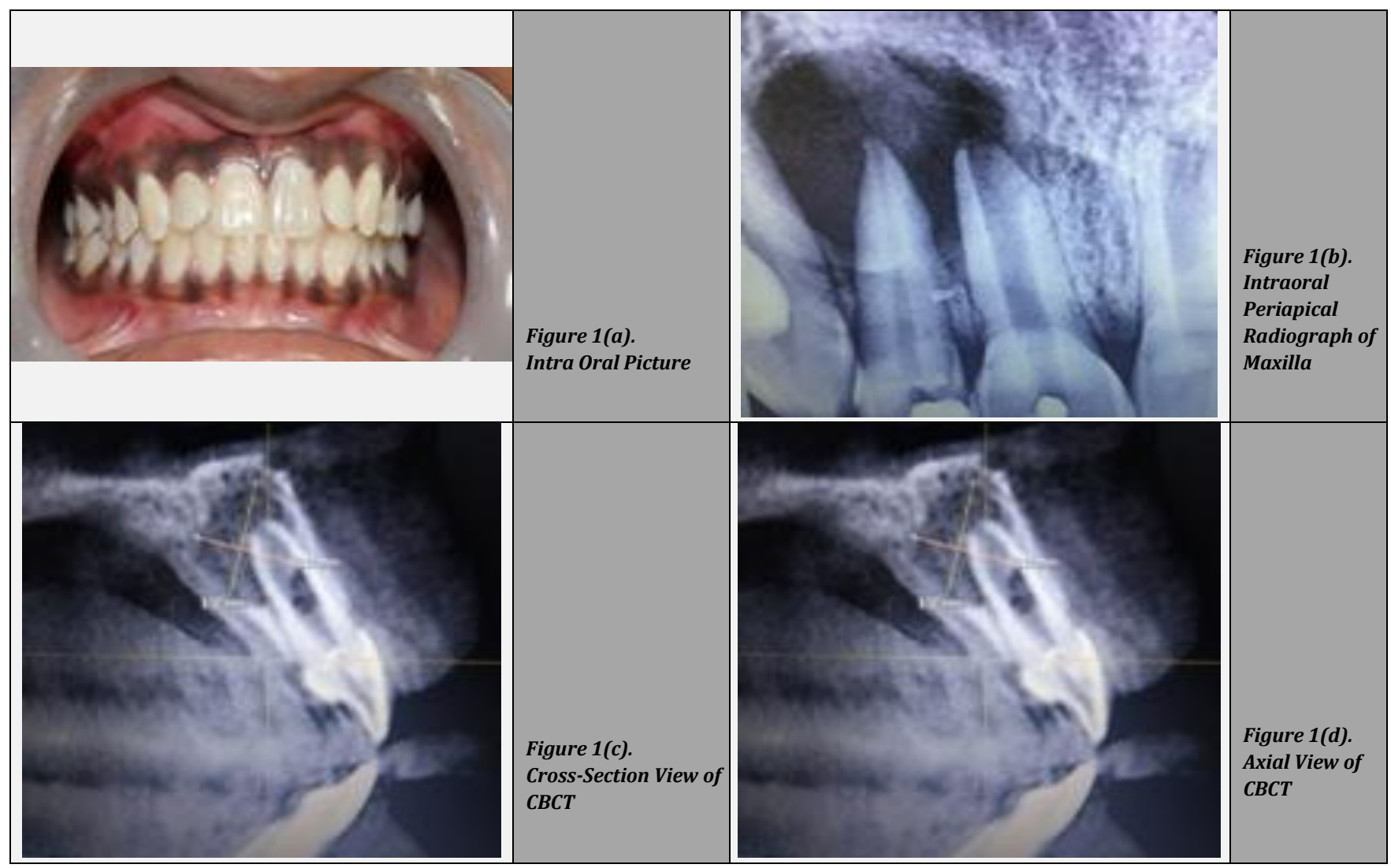



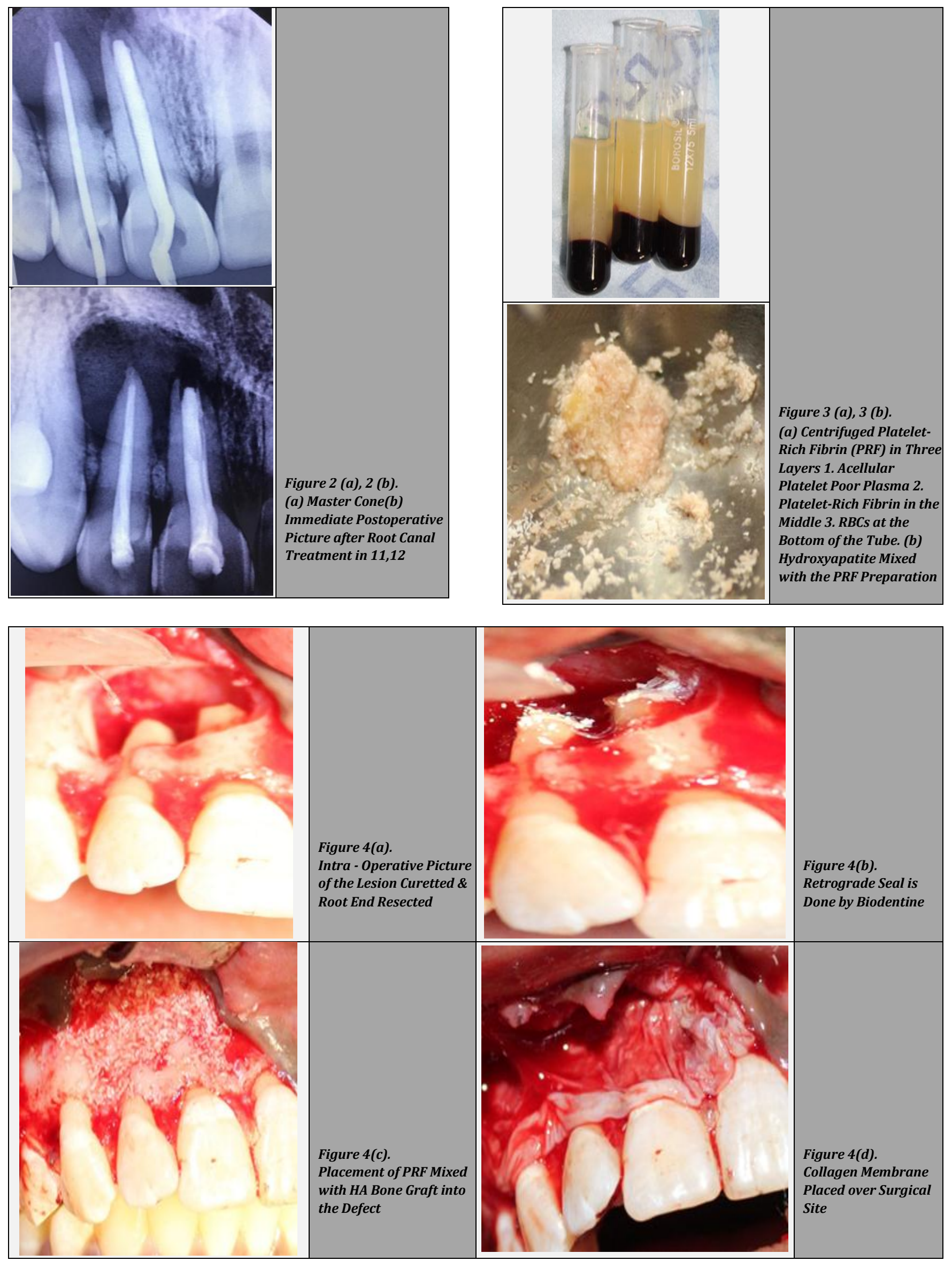

Platelet-rich fibrin (PRF) was prepared using the patient's own blood collected in a $10 \mathrm{ml}$ test tube without anticoagulant, it was then centrifuged for $12 \mathrm{~min}$ at $475.54 \mathrm{~g}$. After it was centrifuged the test tube consisted of three layers 1 . Acellular platelet poor plasma in the upper most part of the tube 2 . Platelet-rich fibrin in the middle 3. RBCs at the bottom of the tube. (Fig 3a) Hydroxyapatite $0.5 \mathrm{mg}$ of pore size of $0.4-0.9$ $\mathrm{mm}$ and a stomatal rate of $15 \%$ was mixed with the PRF 
preparation (Fig 3b). The mixture of PRF - HA was placed in the periapical defect (Fig 4c). Suturing was done with 4 - 0 silk sutures. Patient was prescribed with analgesics and antibiotics and was advised to use chlorhexidine mouth wash. Patient was recalled after 1 week and the suture was removed. On examination of the surgical site wound healing was satisfactory. The patient was reviewed every 2 months. After 1 year follow up, periapical radiograph was taken to confirm the evidence of healing of the periapical bone (Fig 5).

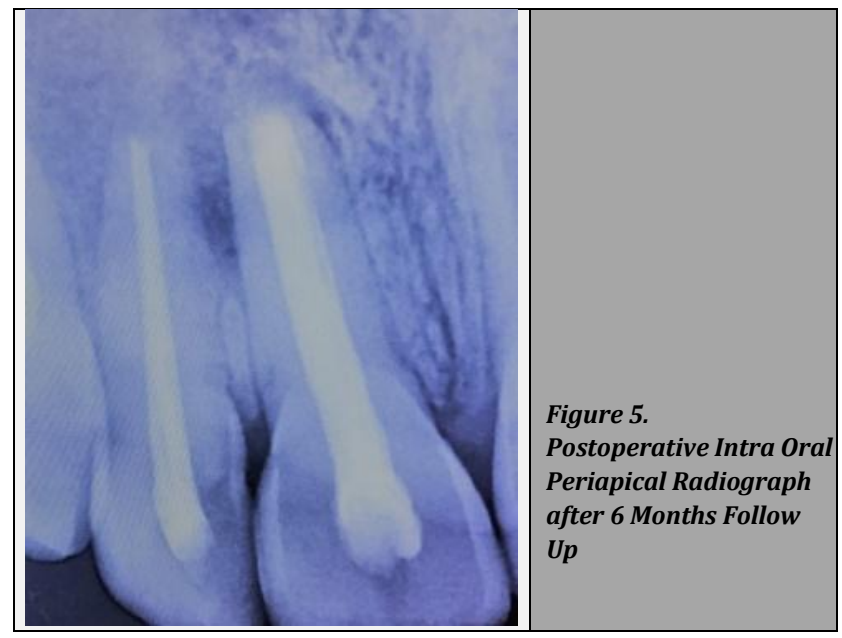

\section{Case Report 2}

A young 24-year-old male patient reported to the Department of Conservative Dentistry and Endodontics, with the chief complaint of pain and pus discharge from the gums of the upper front tooth for the past 4 - 5 months. History revealed that he had a fall and hurt his maxillary anterior teeth 3 years back, which resulted in fracture of his maxillary anterior teeth and he had undergone treatment for the same 3 years back. Intraoral examination revealed an infected sinus in relation to 21 with pus discharge, pain on percussion of 21,22 . Grade II mobility in 21 and Grade I mobility in 22 were seen. A nonvital response was elicited from 21, 22 using thermal and electric pulp tester. Intraoral periapical radiograph revealed that 21,22 were root canal treated. An oval periapical radiolucency about $3 \times 2.5 \mathrm{~cm}$ with well-defined borders was seen in the periapical area extending to the apical one-third of roots of 21, 22. (Fig. 6a).

Surgical intervention was planned, basic blood investigations were carried out and the results were within physiological limits. Informed consent was also obtained from the patient. A day prior to the surgery the patient was administered prophylactic antibiotics.

Sulcular incision starting from distal aspect of 11 up to 23 under local anaesthesia was made and a full-thickness mucoperiosteal flap was raised. The surgical site revealed complete loss of cortical plate in relation to 21 and 22 (fig. 6b). Curettage of the periapical defect including the cystic lining was done. Retrograde filling was done using Biodentine (Fig. 6 c).PRF with hydroxyapatite bone graft was compacted into the periapical bony defect (Fig. 6 d). Suturing was done. The patient was recalled after 1 week, suture was removed. IOPA radiographs were taken every 2 months. IOPA revealed increasing levels of radiopacity proving the uptake of graft and periapical bone formation (Fig. 6 e).

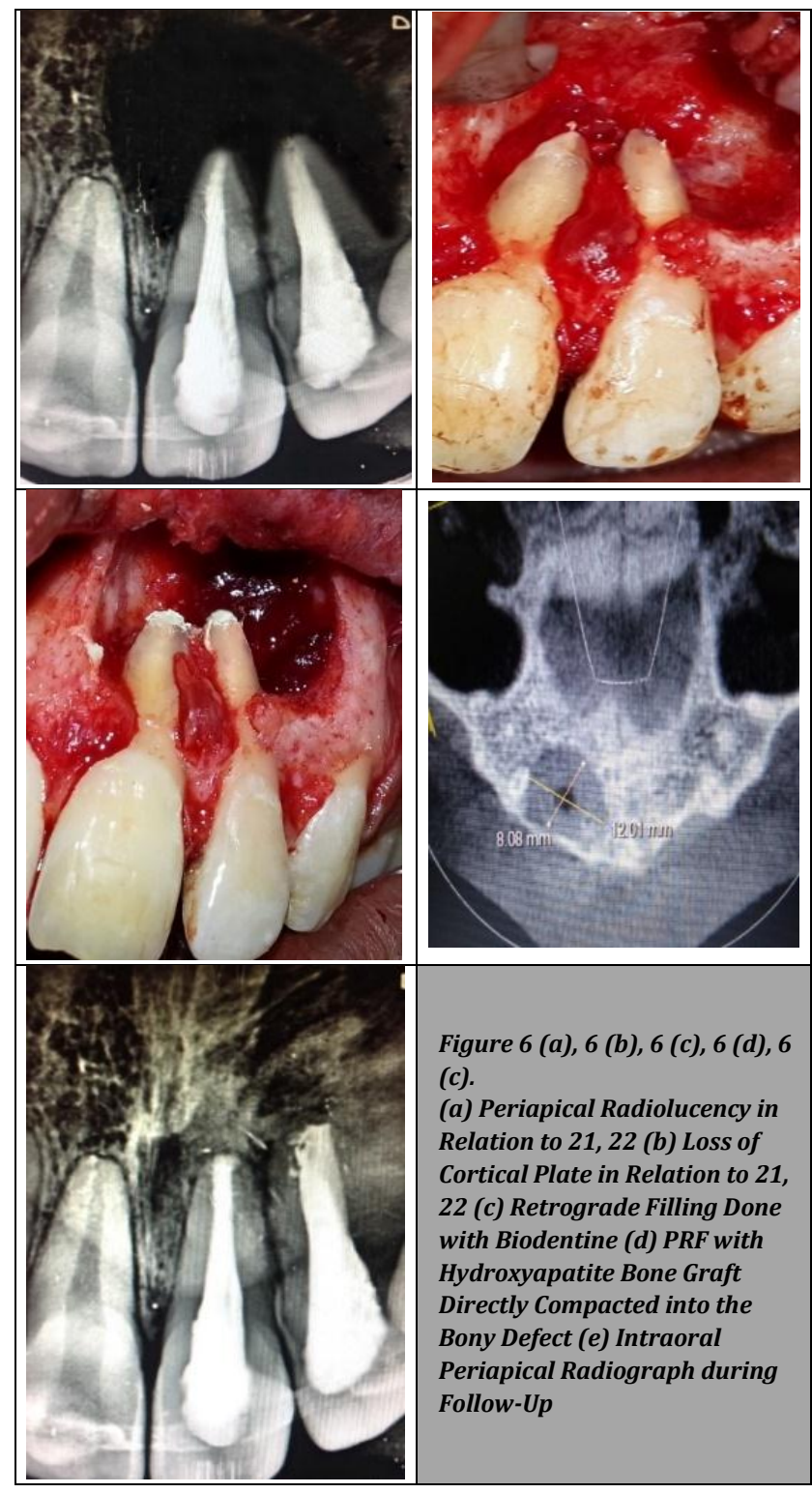

\section{DISCUSSION}

Periapical lesion is inflammation around the apex of the tooth which occurs due to spread of infection following pulpal necrosis, dental trauma or overextended obturation. Orthograde root canal therapy is the first treatment option and has reported $85 \%$ of success rate. In clinical situations where nonsurgical treatment cannot solve the problem or when the treatment fails, periapical surgery is the last resort. ${ }^{3}$ The main objective of periapical surgery is to resolve periapical lesion and favour regeneration.

After the surgical intervention, healing usually occurs by repair or regeneration. ${ }^{2}$ Regenerative surgery using barrier membrane and graft material aid in the formation of new tissues and functional reconstruction. The critical factors essential for bone regeneration after the surgical intervention are primary wound closure, angiogenesis, source of undifferentiated mesenchymal cells, space maintenance, and stability of the wound [ PASS principle]. ${ }^{5}$

The present case reported the clinical efficacy of platelet rich fibrin (PRF) and hydroxyapatite (HA) in the treatment of periapical bony defect. Pradeep et al. stated that regenerative 
effect of PRF is increased when it is combined with HA in the treatment of intra-bony defects. ${ }^{6}$ The main component of PRF are the growth factors which are very essential for wound healing. PRF contains platelet derived growth factor (PDGF), TGF $\beta 1$ and $\beta 2$, insulin like growth factor (IGF), epidermal growth factor (EGF), vascular EGF, and fibroblast growth factors which play a major role in bone metabolism and regulates cell proliferation. PDGF activates collagenase which improves the strength of healed tissue. TGF $\beta$ activates fibroblasts to form procollagen and it deposits collagen. PRF fastens healing by controlling the local inflammatory response. ${ }^{3}$

Platelet rich fibrin when used with HA bone grafts improves the handling properties of graft materials. 7,8 PRF acts a matrix and the integrity of the bone graft material is maintained, it brings about revascularization between the bone graft particles by neoangiogenesis. Hydroxyapatite bone grafting material is porous with bone-conductive property which allows the osteogenic cells from existing bone surfaces to outgrow into periapical defect where the bone graft was placed. HA does not contain any organic components so, does not induce any allergic reaction and is well tolerated clinically. ${ }^{9}$

Advantages of using of PRF with HA bone graft are ${ }^{10}$

1. The fibrin clot acts as a biological - connector between the bone particles.

2. The fibrin network in the defective site facilitates cellular migration for effective regeneration, neoangiogenesis, vascularisation resulting in graft survival.

3. The platelet cytokines (PDGF, TGF $\alpha$, IGF1) are gradually released as the fibrin matrix is resorbed, resulting in healing. ${ }^{11}$

4. The presence of leukocytes and cytokines in the fibrin network renders itself self-regulation of inflammatory and infection within the grafted material.

5. As PRF is obtained from patient's own blood, there are less chances of infectious disease transmission.

6. PRF harvesting is economical

An important factor for the success of periapical surgery is placing a biocompatible seal or root end filling which helps in repair and regeneration of periapical hard and soft tissues. In this case report Biodentine was used as the retrograde filling material.

Biodentine is a calcium-silicate based material which is biocompatible and easy to handle with short setting time, it is claimed to be a bioactive dentin substitute which is effective in management of root perforations, apexification and retrograde filling. ${ }^{7}$

\section{CONCLUSIONS}

Within the plasma rich fibrin there is a slow conversion of fibrinogen into fibrin as small quantities of thrombin are physiologically available in the blood sample. Thus, there is a physiologic architecture favourable for the healing process. In the reported clinical cases treated with surgical intervention, large periapical lesion was resolved effectively by repair and regeneration using a combination of PRF and HA bone graft and successful outcome of the patient was confirmed radiographically.

Financial or other competing interests: None.

Disclosure forms provided by the authors are available with the full text of this article at jemds.com.

\section{REFERENCES}

[1] Krishna BS, Bukhari SH. Surgical management of a periapical lesion associated with left maxillary central and lateral incisors using platelet-rich fibrin: a case report. European Journal of Biomedical and Pharmaceutical Sciences 2020;7(1):449-53.

[2] Shivashankar VY, Johns DA, Vidyanath S, et al. Combination of platelet rich fibrin, hydroxyapatite and PRF membrane in the management of large inflammatory periapical lesion. J Conserv Dent 2013;16(3):261-4.

[3] Prasanthi NNVD, Chittem J, Simpsy GS, et al. Surgical management of a large inflammatory periapical lesion with platelet-rich fibrin. J Interdiscip Dent 2017;7(2):769.

[4] Kattimani VS, Kondaka S. Hydroxyapatite-past, present and future in bone regeneration. Bone and Tissue Regeneration Insights 2016;7:9-19.

[5] Boyapati L, Wang Hl. The role of stress in periodontal disease and wound healing. Periodontol 2000 2007;44:195-210.

[6] Pradeep AR, Bajaj P, Rao NS, et al. Platelet-rich fibrin combined with a porous hydroxyapatite graft for the treatment of three-wall intrabony defects in chronic periodontitis: a randomized controlled clinical trial. J Periodontol 2012;83(12):1499-507.

[7] Hiremath $H$, Motiwala $T$, Jain $P$, et al. Use of secondgeneration platelet concentrate (platelet-rich fibrin) and hydroxyapatite in the management of large periapical inflammatory lesion: a computed tomography scan analysis. Indian J Dent Res 2014;25(4):517-20.

[8] Kaigler D, Cirelli JA, Giannobile WV. Growth factor delivery for oral and periodontal tissue engineering. Expert Opin Drug Deliv 2006;3(5):647-62.

[9] Hiremath H, Gada N, Kini Y. Single-step apical barrier placement in immature teeth using mineral trioxide aggregate and management of periapical inflammatory lesion using platelet-rich plasma and hydroxyapatite. J Endod 2008;34(8):1020-4.

[10] Simonpieri A, Del Corso M, Sammartino G, et al. The relevance of Choukroun's platelet-rich fibrin and metronidazole during complex maxillary rehabilitations using bone allograft. Part II: implant surgery, prosthodontics and survival. Implant Dent 2009;18(3):220-9.

[11] Rajakeerthi R, Nivedhitha MS. Interdisciplinary management of large Periapical lesion with endodontic origin-a short review. J Adv Pharm Edu Res 2018;8(3):4952. 